\title{
Beneficios y riesgos de la hormona del crecimiento en adultos con déficit de la misma
}

\author{
Benefits and risks of growth hormone in adults with growth hormone deficiency
}

\author{
Juan J. Díez, Fernando Cordido
}

\begin{abstract}
Resumen
La deficiencia de hormona del crecimiento («growth hormone», GH) en el adulto es un síndrome clínico plenamente reconocido que entraña consecuencias adversas para la salud. Muchas de ellas pueden ser mejoradas mediante el tratamiento con GH recombinante. Este tratamiento induce un aumento de la masa magra y una reducción de la masa adiposa. En estudios a largo plazo la densidad mineral ósea se incrementa y mejora la fuerza muscular. La calidad de vida relacionada con la salud suele incrementarse. El perfil lipídico y algunos marcadores de riesgo cardiovascular mejoran con el tratamiento. Este, sin embargo, no está exento de riesgos. La GH eleva la glucemia, el índice de masa corporal y la circunferencia de la cintura, y puede favorecer el desarrollo a largo plazo de diabetes y de síndrome metabólico, según algunos estudios. El riesgo de neoplasia no parece incrementado en adultos tratados con GH, pero existen algunos subgrupos de riesgo elevado. Las deficiencias metodológicas y las dificultades inherentes a los estudios a largo plazo impiden extraer conclusiones definitivas sobre la relación entre GH y supervivencia, por lo que la investigación en este campo debe permanecer activa.
\end{abstract}

\begin{abstract}
Adult growth hormone (GH) deficiency is a well-recognized clinical syndrome with adverse health consequences. Many of these may improve after replacement therapy with recombinant GH. This treatment induces an increase in lean body mass and a decrease in fat mass. In long-term studies, bone mineral density increases and muscle strength improves. Health-related quality of life tends to increase after treatment with GH. Lipid profile and markers of cardiovascular risk also improve with therapy. Nevertheless, GH replacement therapy is not without risk. According to some studies, GH increases blood glucose, body mass index and waist circumference and may promote long-term development of diabetes and metabolic syndrome. Risk of neoplasia does not appear to be increased in adults treated with GH, but there are some high-risk subgroups. Methodological shortcomings and difficulties inherent to long-term studies prevent definitive conclusions about the relationship between GH and survival. Therefore, research in this field should remain active.
\end{abstract}

Palabras clave

Hormona del crecimiento; Deficiencia de hormona del crecimiento; Hipopituitarismo; Tratamiento

Keywords

Growth hormone; Growth hormone deficiency; Hypopituitarism; Treatment

\section{Introducción}

El síndrome de la deficiencia de hormona del crecimiento («growth hormone», GH) en la edad adulta (DGHA) está plenamente definido1 and 2 y se caracteriza por alteraciones en la composición corporal, disminución de la capacidad para el ejercicio y de la calidad de vida, así como por una serie de cambios desfavorables de la función cardiovascular y del metabolismo lipídico e hidrocarbonado. Su diagnóstico se basa en la combinación de enfermedad hipofisaria, hipopituitarismo y disminución de la concentración de factor de crecimiento insulínico tipo 1 («insulin-like growth factor 1», IGF-1), o en respuestas disminuidas de GH a estímulos. Una concentración de IGF-1 por debajo de la normalidad para la edad y sexo en un paciente con afectación de 3 o más ejes hipofisarios es diagnóstico de DGHA. El consenso de expertos define la DGHA grave como un pico de respuesta de GH tras hipoglucemia insulínica inferior a $3 \mu \mathrm{g} / \mathrm{l}$. Este es un límite arbitrario que depende de la metodología del análisis, el laboratorio que lo realiza 
y el tipo de estímulo. Otros estímulos utilizados han sido la hormona liberadora de GH («growth hormone-releasing hormone», GHRH), más arginina, GHRH más péptido 6 liberador de GH o glucagón3 and 4.

Desde 1985 disponemos de GH humana recombinante para el tratamiento de estos pacientes, y desde 1997 su empleo está autorizado en España para el tratamiento de adultos deficitarios. La experiencia acumulada desde entonces es extensa, y hoy en día no hay dudas de que el tratamiento mejora o revierte la mayoría de los signos y síntomas de esta deficiencia hormonal (tabla 1). Sin embargo, este tratamiento hormonal sustitutivo no está exento de riesgos potenciales (tabla 1). En este artículo se revisan los datos más recientes y se resume nuestro conocimiento actual sobre los beneficios y los riesgos que conlleva el tratamiento sustitutivo con $\mathrm{GH}$ en adultos deficitarios de esta hormona.

Tabla 1. Beneficios y riesgos del tratamiento a largo plazo con hormona del crecimiento en pacientes adultos deficitarios

\begin{tabular}{ll}
\hline Beneficios & Riesgos o inconvenientes
\end{tabular}

Composición corporal

Reducción de la masa grasa

Aumento de la masa magra

Aumento de la fuerza muscular

Metabolismo óseo

Aumento de la DMO

Calidad de vida relacionada con la salud

Mejoría en los cuestionarios de calidad de vida
Aumento del IMC

Aumento de la circunferencia de la cintura

Aumento del índice cintura-cadera

Efecto sobre la incidencia de fracturas no demostrado

No mejoría en todas las dimensiones de los cuestionarios de calidad de vida

Mayor beneficio en pacientes con baja calidad de vida al inicio

Probable ausencia de efecto en pacientes con calidad de vida normal

Marcadores de riesgo cardiovascular

Aumento de colesterol HDL

Reducción de la sensibilidad a la insulina

Reducción de colesterol total y LDL

Aumento de glucemia e insulinemia

Reducción de la presión arterial diastólica

Tendencia al aumento de la prevalencia de síndrome metabólico

Reducción de PCR

Aumento de lipoproteína (a)

Reducción del grosor íntima-media carotídeo

Enfermedad cardiovascular Reducción en la tasa de incidencia de infarto de Tendencia a aumento de enfermedad cerebrovascular
miocardio

Neoplasias

No aumento en la tasa de recidiva o progresión de Tendencia a aumento de riesgo de segunda neoplasia en supervivientes de tumores hipotálamo-hipofisarios cáncer infantil tratados con $\mathrm{GH}$ en la infancia

No aumento en el riesgo global de neoplasia en adultos Existen subgrupos con aumento del riesgo de ciertas neoplasias en adultos con DGHA que fueron tratados con $\mathrm{GH}$ en la infancia

Mortalidad

Tendencia a la disminución de la mortalidad global y Persistencia de mayor mortalidad que la población general en algunos cardiovascular del hipopituitarismo estudios

Colesterol HDL: colesterol unido a high density lipoproteins («lipoproteínas de densidad alta»); Colesterol LDL: colesterol unido a low density lipoproteins («lipoproteínas de densidad baja»); DGHA: síndrome de la deficiencia de hormona del crecimiento de comienzo en la edad adulta; DMO: densidad mineral ósea; GH: growth hormone («hormona del crecimiento»); IMC: índice de masa corporal; PCR: proteína C reactiva. 


\section{Beneficios del tratamiento con hormona del crecimiento}

El tratamiento con GH se asocia con efectos beneficiosos sobre la composición corporal, la estructura ósea, la calidad de vida relacionada con la salud y diversos factores de riesgo cardiovascular ${ }^{5}$ and 6 .

\section{Composición corporal}

El tratamiento con GH ejerce efectos beneficiosos sobre la composición corporal, induciendo disminución de la masa grasa y aumento de la masa magra. Estos cambios beneficiosos ya se describieron en los primeros estudios realizados ${ }^{7}$. En el de Elbornsson et al. ${ }^{8}$, de 156 pacientes con DGHA, se demuestra una mejoría de la masa magra mantenida durante 15 años, y una marcada disminución inicial de la masa grasa, seguida de un incremento lentamente progresivo a lo largo de los años, posiblemente mediado en parte por el envejecimiento. Son especialmente interesantes los datos del estudio de Filipsson Nyström et al. ${ }^{9}$, que demuestran cómo tras más de 3 años de tratamiento con $\mathrm{GH}$, la suspensión del tratamiento durante 4 meses incrementa la grasa subcutánea y visceral abdominal y disminuye la masa muscular del muslo.

El tratamiento con GH incrementa la fuerza muscular y la capacidad para el ejercicio. En el estudio de Gotherstrom et al., el tratamiento durante 10 años de pacientes con DGHA incrementó la fuerza muscular durante la primera mitad del estudio, y posteriormente protegió del declive de la fuerza muscular que se produce con la edad, provocando la práctica normalización de la fuerza muscular ${ }^{10}$.

\section{Estructura ósea}

El tratamiento con GH en pacientes con DGHA es beneficioso sobre el esqueleto e incrementa la densidad mineral ósea (DMO) ${ }^{5,6 \text { and } 11}$. Generalmente el efecto sobre la DMO es mayor a nivel vertebral que femoral y después de 18-24 meses de tratamiento, y la mayoría de los estudios muestran un incremento del $4-10 \%$ de la $\mathrm{DMO}^{12}$. En pacientes con déficit de GH de inicio en la infancia se ha demostrado que la continuación o restitución del tratamiento durante 2 años en pacientes que completaron el crecimiento induce un incremento significativo de la DMO respecto a los pacientes no tratados ${ }^{13}$. Por tanto, se recomienda la continuación del tratamiento con GH durante el período de transición de la infancia a la edad adulta para obtener una maduración ósea completa.

\section{Calidad de vida}

El tratamiento con GH mejora la calidad de vida relacionada con la salud de la mayoría de los pacientes. La mayor parte de la mejoría de la calidad de vida se produce durante el primer año de tratamiento, aunque este efecto beneficioso persiste a medio y largo plazo ${ }^{14}$. Un grupo especial lo constituyen los pacientes con historia previa de acromegalia que presentan posteriormente una DGHA. Este grupo particular de pacientes presenta un importante deterioro de la calidad de vida relacionada con la salud, que se ve mejorada mediante el tratamiento con $\mathrm{GH}^{15}$.

\section{Marcadores de riesgo cardiovascular}

El tratamiento con GH de pacientes con DGHA mejora diversos factores de riesgo cardiovascular, tales como el perfil lipídico, la función endotelial y los marcadores inflamatorios cardiovasculares. Además, algunos estudios han mostrado mejoras en el grosor de la íntima-media carotídeo y aspectos de la función miocárdica ${ }^{16}$.

La mayoría de los estudios han demostrado un incremento del colesterol unido a lipoproteínas de densidad alta y una disminución del colesterol total y unido a lipoproteínas de densidad baja («low density lipoproteins», LDL), tras la administración de $\mathrm{GH}^{17}$. La suspensión del tratamiento con $\mathrm{GH}$ durante 4 meses, tras más de 3 años de su administración, se acompañó de un incremento del colesterol total y $\mathrm{LDL}^{9}$, lo que muestra de forma indirecta uno de los grandes beneficios del tratamiento con GH en pacientes con DGHA.

En relación con el efecto del tratamiento con GH sobre la presión arterial, en un metaanálisis de los estudios controlados con placebo se demuestra una ligera disminución de la presión arterial diastólica ${ }^{18}$. Los marcadores inflamatorios están elevados en los pacientes con DGHA, y el tratamiento con GH disminuye la proteína $\mathrm{C}$ reactiva, y su suspensión la incrementa ${ }^{9}$. 
En estudios epidemiológicos el incremento del grosor de la íntima-media carotídea es un importante predictor de enfermedad coronaria. El tratamiento con GH en los pacientes con DGHA disminuye este parámetro $^{16}$. Es especialmente interesante el estudio de Makimura et al. ${ }^{19}$, en el que en un modelo clínico similar, la obesidad con disminución de la secreción de $\mathrm{GH}$, la administración de forma aleatorizada y controlada con placebo de un análogo de GHRH indujo, además de una elevación moderada de IGF-1, una disminución de la grasa adiposa visceral y del grosor de la íntima-media carotídeo ${ }^{19}$.

\section{Otros efectos beneficiosos}

En los pacientes con DGHA se han observado alteraciones del sueño. Recientemente, Morselli et al. ${ }^{20}$ han demostrado que 4 meses de tratamiento con $\mathrm{GH}$ en este grupo de pacientes revierten las alteraciones en el sueño presentes en los pacientes no tratados; en concreto, se apreció una reducción en la intensidad del sueño de ondas lentas (delta) ${ }^{20}$.

Estudios retrospectivos ${ }^{21}$ and 22 y prospectivos ${ }^{23}$ han mostrado que los pacientes con hipopituitarismo tratados mediante sustitución hormonal habitual, pero sin tratamiento con GH, presentan una mortalidad incrementada en comparación con la población general, especialmente por enfermedad cardiovascular. En un grupo de 1.411 pacientes con hipopituitarismo sin tratamiento con GH, Svensson et al. ${ }^{22}$ hallaron que la mortalidad global y las tasas de infarto de miocardio, episodios cerebrovasculares y neoplasias se encontraban incrementadas en comparación con las de la población general. En una cohorte de 289 pacientes con hipopituitarismo tratados con $\mathrm{GH}$, la mortalidad global fue similar, y la tasa de infarto de miocardio fue inferior a la de la población general, si bien los autores llaman la atención sobre una tendencia hacia un incremento en la tasa de episodios cerebrovasculares en pacientes tratados con $\mathrm{GH}$. Se ha planteado que la sustitución con GH parece proporcionar protección frente al infarto de miocardio ${ }^{22}$.

\section{Riesgos del tratamiento con hormona del crecimiento}

\section{Composición corporal}

No todos los cambios en la composición corporal inducidos por la GH son favorables. La cintura y el índice de masa corporal (IMC) aumentan significativamente en algunos estudios ${ }^{24}$. En una revisión sistemática de 23 estudios prospectivos de tratamiento con GH durante 5-15 años se demuestra un incremento del IMC, de la circunferencia de la cintura y del índice cintura-cadera. Además, aunque el tratamiento con GH se acompaña de un aumento en la masa magra, la mayoría de los estudios no permiten diferenciar entre el agua extracelular y la masa intracelular ${ }^{6}$.

\section{Estructura ósea}

La deficiencia de GH de comienzo infantil se asocia a reducción de masa ósea, pero la de comienzo adulto, no siempre ${ }^{25}$ and 26 . Las mejores respuestas se dan en varones y en pacientes con DMO más baja al comienzo del tratamiento. No obstante, los estudios no detallan el uso de calcio, vitamina D o fármacos antirresortivos $^{6}$, por lo que es difícil extraer conclusiones. Además, hasta la fecha, ningún estudio controlado aleatorizado ha demostrado efectos del tratamiento con GH sobre la tasa de fracturas.

\section{Calidad de vida}

La mejoría de la calidad de vida no se observa ni en todos los adultos ni en todas las dimensiones sociopsicológicas analizadas ${ }^{27}$ and 28 . Algunos estudios han mostrado, incluso, empeoramiento en algunas dimensiones, como la función social y salud mental, en adultos con deficiencia de comienzo infantil ${ }^{29}$. Por lo general, las respuestas son muy heterogéneas y responden mejor los pacientes con calidad de vida más deteriorada antes del tratamiento. Por ello es posible que los pacientes que tienen basalmente una calidad de vida normal no se beneficien del tratamiento con $\mathrm{GH}$ en este aspecto.

\section{Marcadores de riesgo cardiovascular}

En algunos casos se ha demostrado que la lipoproteína (a), un marcador independiente de riesgo cardiovascular, se incrementa significativamente durante el tratamiento con $\mathrm{GH}^{30}$. Además, un estudio ha 
observado un aumento de placas de aterosclerosis tras 6 meses de tratamiento sustitutivo con GH en adultos con deficiencia congénita de GH debida a mutación del receptor de GHRH, no tratados previamente $^{31}$.

\section{Efectos adversos}

Los efectos secundarios derivados de la retención hídrica provocada por la GH se presentan en un 5$18 \%$ de los pacientes. Consisten en edema, artralgias, mialgias, parestesias y síndrome del túnel carpiano, y suelen responder a reducción de dosis. Los ancianos, mujeres y pacientes con sobrepeso presentan mayor riesgo de desarrollo de estos efectos. La retinopatía y la hipertensión intracraneal benigna son complicaciones muy raras del tratamiento con GH. Las necesidades de cortisol y de tiroxina pueden incrementarse en pacientes con panhipopituitarismo ${ }^{32}$. Para minimizar el impacto de los efectos adversos es adecuado comenzar el tratamiento con dosis bajas e ir titulando al alza según respuesta clínica y valores de IGF-1, así como utilizar la GH solo dentro de las indicaciones reconocidas por la autoridad sanitaria $^{33}$ y seguir unas recomendaciones generales, que se recogen en la tabla 2.

Tabla 2. Recomendaciones generales para el tratamiento con hormona del crecimiento en adultos con deficiencia de esta, de comienzo en la edad adulta

Prescribir tratamiento con GH a pacientes adultos con deficiencia grave de esta hormona plenamente demostrada con pruebas diagnósticas adecuadas

Utilizar la dosis mínima efectiva en cada paciente, comenzando por dosis bajas

Utilizar dosis de inicio más bajas en ancianos y diabéticos

Seguir las recomendaciones de las guías nacionales e internacionales para el seguimiento de pacientes adultos con DGHA

Evitar concentraciones de IGF-1 por encima del límite superior de la normalidad para la edad y sexo del paciente

Realizar controles de glucemia, hemoglobina $\mathrm{A}_{1 \mathrm{c}} \mathrm{y}$ perfil lipídico de forma periódica y tras cambio de dosis

Realizar periódicamente resonancia magnética hipofisaria en pacientes con historia previa de tumores del área hipotálamohipofisaria

Vigilar en todo momento la aparición de efectos adversos y comunicarlos al Comité Asesor de tratamiento con GH o a la autoridad sanitaria correspondiente

DGHA: síndrome de la deficiencia de la hormona del crecimiento de comienzo en la edad adulta; GH: growth hormone («hormona de crecimiento»); IGF-1: insulin-like growth factor 1 («factor de crecimiento insulínico tipo 1»).

\section{Riesgo de hiperglucemia y diabetes}

El tratamiento con GH reduce la sensibilidad a la insulina y eleva la glucemia. En un estudio realizado en 90 pacientes en tratamiento con GH se demostró un incremento significativo tanto en las concentraciones de glucosa como en las de hemoglobina $A_{1 c}$. Los cambios fueron evidentes a los 6 meses y persistieron tras 2 años de tratamiento ${ }^{34}$. Estos datos se han visto corroborados por un metaanálisis de 37 ensayos clínicos ciegos, aleatorizados y controlados con placebo, que demostró que el tratamiento con GH se acompañaba de un incremento de la glucemia basal y de la concentración de insulina, con independencia de la dosis y duración del tratamiento ${ }^{18}$.

En un ensayo clínico aleatorizado, doble ciego, controlado con placebo, en 166 pacientes adultos deficitarios, el tratamiento con GH se asoció a un empeoramiento de la tolerancia a la glucosa, con aparición de diabetes en el $4 \%$ y de intolerancia hidrocarbonada en el $20 \%$ de los pacientes al cabo de 12 meses, mientras que en el grupo placebo solo un $8 \%$ desarrollaron intolerancia ${ }^{17}$.

En el Hypopituitary Control and Complications Study (HypoCCS) se ha analizado también la prevalencia e incidencia de diabetes en adultos en tratamiento con $\mathrm{GH}^{35}$. Los resultados de un análisis de 2.922 pacientes en EE. UU. y 3.709 pacientes europeos, con un seguimiento medio de 4,1 años, mostró que, en EE. UU., la tasa de incidencia de diabetes ajustada a edad, sexo e IMC es superior en los pacientes tratados con GH que en la población general. En Francia y Alemania las tasas de incidencia fueron comparables con las de la población de referencia, mientras que en Suecia la tasa de incidencia fue de más del doble en pacientes en tratamiento con $\mathrm{GH}$. 
Un análisis más reciente de 5.143 pacientes del estudio Pfizer International Metabolic Database (KIMS), con 20.106 pacientes-año de seguimiento, puso de manifiesto que, en el sur de Suecia, la razón de casos observados/casos esperados fue de 10,8 el primer año de tratamiento con GH, y descendió a 1,9 tras 8 años de este, pero siempre se mantuvo por encima de la unidad. Cuando la incidencia de diabetes de los pacientes en el estudio KIMS se comparó con las tasas de incidencia en poblaciones ajustadas para edad de otras regiones europeas y de EE. UU., las razones casos observados/esperados oscilaron entre 2,11 y $5,22^{36}$.

\section{Riesgo de neoplasia}

Los primeros estudios realizados en niños tratados con $\mathrm{GH}$ extractiva demostraron un mayor riesgo de mortalidad por cáncer $\mathrm{y}$, en particular, por cáncer colorrectal y linfoma de Hodgkin ${ }^{37}$. El riesgo oncogénico durante el tratamiento en niños tratados con GH se estableció en los estudios National Cooperative Growth Study y Pfizer International Growth Database, que evaluaron más de 50.000 pacientes cada uno, con casi 200.000 pacientes-año cada uno. La tasa de incidencia estandarizada de cáncer fue de 1,12 (intervalo de confianza del 95\% [IC 95\%] 0,75-1,61) en el primero de ellos ${ }^{38}$ y de 1,26 (IC 95\% 0,86-1,78) en el segundo ${ }^{39}$, es decir, no se observó un aumento significativo del riesgo de cáncer en estos niños. Tampoco se obtuvo un mayor riesgo de leucemia que en la población general en estos estudios.

El riesgo de segunda neoplasia en niños supervivientes de cáncer se ha analizado en profundidad en el Childhood Cancer Survivor Study. En su escrutinio, realizado en 2002, este estudio demostró que los niños tratados con GH presentan un riesgo relativo de cáncer de 3,21 (IC 95\% 1,88-5,46) frente a los supervivientes de cáncer no tratados con esta hormona ${ }^{40}$. Este riesgo relativo es aún más elevado, de 4,98 (IC 95\% 1,95-12,74), en los supervivientes de leucemia. En el análisis de $2006^{41}$, incluyendo 32 meses más de seguimiento, el riesgo de segunda neoplasia se redujo a 2,15 (IC 95\% 1,33-3,47), pero continuó siendo significativo.

Los datos disponibles en pacientes adultos evidencian que el tratamiento sustitutivo con GH no incrementa la tasa de recidiva o de progresión de los tumores del área hipotálamo-hipofisaria ${ }^{42}$. Sin embargo, estudios de reciente publicación han sembrado dudas sobre el riesgo de tumores en pacientes tratados con GH. El Safety and Appropriateness of Growth hormone treatment in Europe (SAGhE) es un estudio de cohorte de base poblacional que analiza la mortalidad a largo plazo de adultos que fueron tratados con GH durante la infancia, y que incluyó 6.928 pacientes tratados por deficiencia de GH idiopática, disfunción neurosecretora, talla baja idiopática y pequeños para edad gestacional, es decir, etiologías sin riesgo incrementado de neoplasia. El tiempo medio de seguimiento tras finalizar el tratamiento fue de 7,8 años ${ }^{43}$. En este estudio, la tasa estandarizada de mortalidad por neoplasias de hueso y cartílago alcanzó la llamativa cifra de 5,00 (IC 95\% 1,01-14,61). En un grupo de 6.840 adultos incluidos en el estudio HypoCCS, la tasa de incidencia de neoplasia fue de 0,88 (IC 95\% 0,74-1,04), pero ascendió a un 3,79 (IC 95\% 1,39-8,26) cuando se analizaron los pacientes menores de 35 años, y a 2,74 (IC $95 \%$ 1,18-5,42) cuando se analizaron los pacientes de comienzo infantil ${ }^{44}$. Es decir, el riesgo global de cáncer primario en la vida adulta no está incrementado, sin embargo, existen subgrupos con riesgo elevado.

\section{Riesgo de mortalidad}

Un estudio realizado con datos del Registro Nacional Holandés de tratamiento con $\mathrm{GH}^{45}$ comparó 2.229 pacientes en tratamiento con GH con un grupo de control primario de 109 pacientes diagnosticados, pero no tratados, con GH, y un grupo de control secundario de 356 pacientes tratados con GH en los que el tratamiento se había suspendido. La tasa de mortalidad estandarizada frente a población general fue de 1,27 (IC 95\% 1,04-1,56) para el grupo de tratamiento. Esta tasa descendió a 1,29 (IC 95\% 1,05-1,59) cuando se excluyeron pacientes con acromegalia y enfermedad de Cushing, y a 1,00 (IC 95\% 0,79-1,26) tras la exclusión de pacientes de alto riesgo (craneofaringioma y otras causas). Destaca que los autores no hallaron un aumento significativo de mortalidad en los 2 grupos controles no tratados. Se encontró, además, un aumento significativo de la tasa estandarizada de mortalidad por enfermedad cardiovascular en mujeres del grupo de tratamiento (2,52 [IC 95\% 1,57-4,06]), que persistió tras la exclusión de los pacientes de alto riesgo.

Una evaluación de la base de datos KIMS realizada en $2008^{46}$, que incluyó 4.486 pacientes y 17.573 pacientes-año de tratamiento con GH, concluyó que la tasa estandarizada de mortalidad en estos pacientes era de 1,05 (IC 95\% 0,88-1,24), mientras que la tasa estandarizada de mortalidad cardiovascular era de 
0,98 (IC 95\% 0,70-1,30), es decir, que el tratamiento con GH podría reducir la elevada mortalidad global y cardiovascular que caracteriza a los pacientes con hipopituitarismo. Sin embargo, estos datos no se han confirmado en un análisis posterior del mismo estudio $\mathrm{KIMS}^{47}$. La evaluación de un grupo de 13.983 pacientes tratados con $\mathrm{GH}$, con una media de seguimiento de 4,9 años, mostró que la mortalidad por todas las causas en estos pacientes fue un $13 \%$ superior a la de la población general (tasa estandarizada de mortalidad de 1,13 [IC 95\% 1,04-1,24]). No se observó un incremento de la mortalidad por enfermedad cardiovascular o por cáncer.

En el ya mencionado estudio $\mathrm{SAGhE}^{43}$, el riesgo de mortalidad global en pacientes que recibieron $\mathrm{GH}$ en edad infantil se incrementó un 33\%. En este estudio, la tasa estandarizada de mortalidad por enfermedades circulatorias fue de 3,07 (IC 95\% 1,40-5,83), y ascendió a 5,29 (IC 95\% 1,42-13,55) y a 6,66 (IC 95\% 1,79-17,05) cuando se consideraron las muertes por enfermedad cerebrovascular y por hemorragia intracraneal, respectivamente.

En resumen, como reconoce la guía de la Endocrine Society ${ }^{5}$, aunque la mortalidad se halla incrementada en pacientes con hipopituitarismo y la deficiencia de GH se ha implicado en ello, no existen datos que demuestren que el tratamiento con GH mejore la supervivencia de los pacientes.

\section{Conclusiones y visión de futuro}

La GH ejerce incuestionables efectos beneficiosos en muchos pacientes con DGHA. Es necesario identificar los candidatos potenciales de tratamiento y confirmar el DGHA antes del inicio del mismo. La $\mathrm{GH}$ ha demostrado efectos beneficiosos sobre la composición corporal, la capacidad para el ejercicio, la estructura ósea, los lípidos y la calidad de vida (tabla 1). La dosis debe ser cuidadosamente individualizada para evitar los efectos secundarios, y es necesaria una vigilancia periódica adecuada (tabla 2). No se ha demostrado que el tratamiento con GH mejore la mortalidad global, las fracturas óseas, la enfermedad cardiaca clínica o el cáncer.

Hay que ser prudente en la interpretación de los resultados de los estudios publicados, incluso con los procedentes de las grandes bases de datos internacionales, ya que todos presentan deficiencias metodológicas y han de ser valorados con cautela. Los estudios son heterogéneos y con diseños variables, y muchos de ellos carecen de grupo control. A largo plazo no existen estudios controlados con placebo. Además, existen factores de confusión que influyen en los resultados, tales como la radiación, la quimioterapia y los tratamientos concomitantes. En ocasiones, el seguimiento es escaso para llegar a conclusiones sobre neoplasias y mortalidad. No obstante, si bien nuestro conocimiento sobre el tratamiento sustitutivo con $\mathrm{GH}$ es aún imperfecto, los recientes estudios que hemos revisado en este artículo nos permiten dar respuestas basadas en la evidencia científica a algunas preguntas de relevancia clínica (tabla 3).

Tabla 3. Preguntas y respuestas sobre el tratamiento con hormona del crecimiento en adultos deficitarios

Pregunta $\quad$ Mejor respuesta disponible

¿El tratamiento con GH mejora la calidad de vida Sí, en la mayoría de los estudios de los pacientes?

¿Se consigue una mejoría en la composición Sí, reduce la masa adiposa y aumenta la masa magra. A largo plazo, también corporal?

¿El tratamiento con GH disminuye el riesgo Sí, la mayoría de los marcadores de riesgo cardiovascular experimentan un cardiovascular? cambio favorable

¿Se reduce la tasa de fracturas mediante No se ha demostrado tratamiento a largo plazo?

¿El tratamiento con GH modifica la glucemia? Sí, a largo plazo las cifras de glucemia se elevan y en algunos casos aumenta la incidencia de intolerancia hidrocarbonada y diabetes

¿El tratamiento durante la vida adulta aumenta el Probablemente no, pero hay datos que indican que hay subgrupos con mayor riesgo de cáncer? riesgo de neoplasia

¿El tratamiento con GH disminuye la mortalidad No, en los pacientes adultos tratados con GH persiste un incremento de la asociada al hipopituitarismo? mortalidad, en la mayoría de los estudios 
Como en otros aspectos de la enfermedad hipofisaria ${ }^{48}$, es posible mejorar y optimizar el tratamiento de pacientes con DGHA. Existen en desarrollo formas de GH de liberación prolongada, que permitirían reducir la frecuencia de las inyecciones ${ }^{49}$. Si se confirma su eficacia y seguridad, este tipo de modificación posológica sería especialmente bienvenida en la práctica clínica habitual. La posibilidad de modificaciones en la molécula de GH que incrementen sus efectos beneficiosos y disminuyan sus efectos secundarios es una vía de estudio a considerar. La seguridad a largo plazo del tratamiento con GH es una obligación de los profesionales sanitarios, la industria farmacéutica y las autoridades sanitarias, por lo que es preciso un control periódico tanto de los beneficios como de los efectos adversos. Siguen siendo necesarios estudios a largo plazo con variables de resultados relevantes, tales como incidencia de fracturas, enfermedad cardiovascular, cáncer o mortalidad.

\section{Conflicto de intereses}

Los autores declaran no tener ningún conflicto de intereses en relación con este artículo y no tener intereses comerciales ni relación de dependencia profesional con ninguna compañía farmacéutica comercializadora de hormona del crecimiento, ni haber cobrado honorarios por la redacción de este artículo.

\section{Bibliografía}

1. J.J. Díez, A. Gómez-Pan, P. Iglesias. Hacia un concepto del síndrome de deficiencia de hormona del crecimiento en adultos. Med Clin (Barc), 107 (1996), pp. 218-223.

2. J.J. Díez. El síndrome de deficiencia de hormona del crecimiento en adultos: criterios actuales para el diagnóstico y tratamiento. Med Clin (Barc), 114 (2000), pp. 468-477.

3. F. Cordido, P. Alvarez-Castro, M.L. Isidro, F.F. Casanueva, C. Dieguez. Comparison between insulin tolerance test, growth hormone $(\mathrm{GH})$-releasing hormone $(\mathrm{GHRH}), \mathrm{GHRH}$ plus acipimox and GHRH plus GH-releasing peptide- 6 for the diagnosis of adult GH deficiency in normal subjects, obese and hypopituitary patients. Eur J Endocrinol, 149 (2003), pp. 117-122.

4. F. Cordido, A. Peñalva, R. Peino, F.F. Casanueva, C. Dieguez. Effect of combined administration of growth hormone $(\mathrm{GH})$-releasing hormone, GH-releasing peptide-6, and pyridostigmine in normal and obese subjects. Metabolism, 44 (1995), pp. 745-748.

5. M.E. Molitch, D.R. Clemmons, S. Malozowski, G.R. Merriam, M.L. Vance. Evaluation and treatment of adult growth hormone deficiency: An Endocrine Society clinical practice guideline. J Clin Endcrinol Metab, 96 (2011), pp. 1587-1609.

6. N.M. Appelman-Dijkstra, K.M. Claessen, F. Roelfsema, A.M. Pereira, N.R. Biermasz. Long-term effects of recombinant human GH replacement in adults with GH deficiency: A systematic review. Eur J Endocrinol, 169 (2013), pp. R1-R14.

7. F. Salomon, R.C. Cuneo, R. Hesp, P.H. Sonksen. The effects of treatment with recombinant human growth hormone on body composition and metabolism in adults with growth hormone deficiency. N Engl J Med, 321 (1989), pp. 1797-1803.

8. M. Elbornsson, G. Gotherstrom, I. Bosaeus, B.A. Bengtsson, G. Johannsson, J. Svensson. Fifteen years of GH replacement improves body composition and cardiovascular risk factors. Eur J Endocrinol, 168 (2013), pp. 745753.

9. H. Filipsson Nyström, E.J. Barbosa, A.G. Nilsson, L.L. Norrman, O. Ragnarsson, G. Johannsson. Discontinuing long-term GH replacement therapy--A randomized, placebo-controlled crossover trial in adult GH deficiency. J Clin Endocrinol Metab, 97 (2012), pp. 3185-3195.

10. G. Gotherstrom, M. Elbornsson, K. Stibrant-Sunnerhagen, B.A. Bengtsson, G. Johannsson, J. Svensson. Ten years of growth hormone $(\mathrm{GH})$ replacement normalizes muscle strength in GH-deficient adults. J Clin Endocrinol Metab, 94 (2009), pp. 809-816.

11. D. Cabo, A. Lecube, M. Barrios, J. Mesa. Terapia sustitutiva a largo plazo del déficit de hormona del crecimiento en la edad adulta. Med Clin (Barc), 136 (2011), pp. 659-664.

12. B.M. Biller, G. Sesmilo, H.B. Baum, D. Hayden, D. Schoenfeld, A. Klibanski. Withdrawal of long-term physiological growth hormone $(\mathrm{GH})$ administration: Differential effects on bone density and body composition in men with adult-onset GH deficiency. J Clin Endocrinol Metab, 85 (2000), pp. 970-976.

13. L.E. Underwood, K.M. Attie, J. Baptista. Growth hormone (GH) dose-response in young adults with childhoodonset GH deficiency: A two-year, multicenter, multiple-dose, placebo-controlled study. J Clin Endocrinol Metab, 88 (2003), pp. 5273-5280.

14. M. Rosilio, W.F. Blum, D.J. Edwards, E.P. Shavrikova, D. Valle, S.W. Lamberts, et al. Long-term improvement of quality of life during growth hormone $(\mathrm{GH})$ replacement therapy in adults with GH deficiency, as measured by questions on life satisfaction-hypopituitarism (QLS-H). J Clin Endocrinol Metab, 89 (2004), pp. 1684-1693.

15. C. Giavoli, E. Profka, E. Verrua, C.L. Ronchi, E. Ferrante, S. Bergamaschi, et al. GH replacement improves quality of life and metabolic parameters in cured acromegalic patients with growth hormone deficiency. J Clin Endocrinol Metab, 97 (2012), pp. 3983-3988. 
16. J. Gibney, J.D. Wallace, T. Spinks, L. Schnorr, A. Ranicar, R.C. Cuneo, et al. The effects of 10 years of recombinant human growth hormone (GH) in adult GH-deficient patients. J Clin Endocrinol Metab, 84 (1999), pp. 2596-2602.

17. A.R. Hoffman, J.E. Kuntze, J. Baptista, H.B. Baum, G.P. Baumann, B.M. Biller, et al. Growth hormone (GH) replacement therapy in adult-onset gh deficiency: Effects on body composition in men and women in a doubleblind, randomized, placebo-controlled trial. J Clin Endocrinol Metab, 89 (2004), pp. 2048-2056.

18. P. Maison, S. Griffin, M. Nicoue-Beglah, N. Haddad, B. Balkau, P. Chanson. Impact of growth hormone (GH) treatment on cardiovascular risk factors in GH-deficient adults: A metaanalysis of blinded, randomized, placebocontrolled trials. J Clin Endocrinol Metab, 89 (2004), pp. 2192-2199.

19. H. Makimura, M.N. Feldpausch, A.M. Rope, L.C. Hemphill, M. Torriani, H. Lee, et al. Metabolic effects of a growth hormone-releasing factor in obese subjects with reduced growth hormone secretion: A randomized controlled trial. J Clin Endocrinol Metab, 97 (2012), pp. 4769-4779.

20. L.L. Morselli, A. Nedeltcheva, R. Leproult, K. Spiegel, E. Martino, J.J. Legros, et al. Impact of GH replacement therapy on sleep in adult patients with GH deficiency of pituitary origin. Eur J Endocrinol, 168 (2013), pp. $763-$ 770.

21. T. Rosén, B-Å. Bengtsson. Premature mortality due to cardiovascular disease in hypopituitarism. Lancet, 336 (1990), pp. 285-288.

22. J. Svensson, B.A. Bentsson, T. Rosén, A. Odén, G. Johannsson. Malignant disease and cardiovascular morbidity in hypopituitary adults with or without growth hormone replacement therapy. J Clin Endocrinol Metab, 89 (2004), pp. 3306-3312.

23. J. Tomlinson, N. Holden, R. Hills, K. Wheatley, R. Clayton, A. Bates, et al. Association between premature mortality and hypopituitarism. West Midlands Prospective Hypopituitary Study Group. Lancet, 357 (2001), pp. 425-431.

24. K.M. Claessen, N.M. Appelman-Dijkstra, D.M. Adoptie, F. Roelfsema, J.W. Smit, N.R. Biermasz, et al. Metabolic profile in growth hormone-deficient (GHD) adults after long-term recombinant human growth hormone (rhGH) therapy. J Clin Endocrinol Metab, 98 (2013), pp. 352-361.

25. R.D. Murray, B. Columb, J.E. Adams, S.M. Shalet. Low bone mass is an infrequent feature of the adult growth hormone deficiency syndrome in middle-age adults and the elderly. J Clin Endocrinol Metab, 89 (2004), pp. $1124-1130$.

26. A.P. Jørgensen, K.J. Fourgner, T. Ueland, O. Gudmundsen, P. Burman, T. Schreiner, et al. Favorable long-term effects of growth hormone replacement therapy on quality of life, bone metabolism, body composition and lipid levels in patients with adult-onset growth hormone deficiency. Growth Horm IGF Res, 21 (2011), pp. 69-75.

27. M. Koltowska-Häggström, A.F. Mattsson, S.M. Shalet. Assessment of quality of life in adult patients with GH deficiency. KIMS contribution to clinical practice and pharmacoeconomic evaluations. Eur J Endocrinol, 161 (Suppl 1) (2009), pp. S51-S64.

28. J. Mook, C. Albrecht, N. Friedrich, H. Völzke, M. Nauck, M. Koltowska-Häggström, et al. Health-related quality of life and IGF-1 in GH-deficient adult patients on GH replacement therapy: Analysis of the German KIMS data and the Study of Health in Pomerania. Eur J Endocrinol, 160 (2009), pp. 17-24.

29. H. Urushihara, S. Fukuhara, S. Tai, S. Morita, K. Chihara. Heterogeneity in responsiveness of perceived quality of life to body composition changes between adult- and childhood-onset Japanese hypopituitary adults with GH deficiency during GH replacement. Eur J Endocrinol, 156 (2007), pp. 637-645.

30. G. Wiersinga, S.M. Shalet. Changes in lipoprotein (a) levels measured by six kit methods during growth hormone treatment of growth hormone deficient adults. Growth Horm IGF Res, 10 (2000), p. 231.

31. J.L. Oliveira, M.H. Aguiar-Oliveira, A. D’Oliveira, R.M. Pereira, C.R. Oliveira, C.T. Farias, et al. Congenital growth hormone $(\mathrm{GH})$ deficiency and atherosclerosis: Effects of GH replacement in GH-naive adults. J Clin Endocrinol Metab, 92 (2007), pp. 4664-4670.

32. D.M. Cook, K.C. Yuen, B.M. Biller, S.F. Kemp, M.L. Vance, American Association of Clinical Endocrinologists. American Association of Clinical Endocrinologists medical guidelines for clinical practice for growth hormone use in growth hormone-deficient adults and transition patients-2009 update. Endocr Pract, 15 (Suppl 2) (2009), pp. 1-29.

33. Junta Directiva de la Sociedad Española de Endocrinología y Nutrición. Aplicaciones de la hormona del crecimiento en humanos. Med Clin (Barc), 137 (2011), pp. 475-476.

34. D. Florakis, V. Hung, G. Kaltsas, D. Coyte, P.J. Jenkins, S.L. Chew, et al. Sustained reduction in circulating cholesterol in adult hypopituitary patients given low dose titrated growth hormone replacement therapy: A two year study. Clin Endocrinol (Oxf), 53 (2000), pp. 453-459.

35. A.F. Attanasio, H. Jung, D. Mo, P. Chanson, R. Bouillon, K.K. Ho, et al. Prevalence and incidence of diabetes mellitus in adult patients on growth hormone replacement of growth hormone deficiency: A surveillance database analysis. J Clin Endocrinol Metab, 96 (2011), pp. 2255-2261.

36. A. Luger, A.F. Mattsson, M. Koltowska-Häggström, M. Thunander, M. Góth, J. Verhelst, et al. Incidence of diabetes mellitus and evolution of glucose parameters in growth hormone-deficient subjects during growth hormone replacement therapy: A long-term observational study. Diabetes Care, 35 (2012), pp. 57-62.

37. A.J. Swerdlow, C.D. Higgins, P. Adlard, M.A. Preece. Risk of cancer in patients treated with human pituitary growth hormone in the UK, 1959-85: A cohort study. Lancet, 360 (2002), pp. 273-277.

38. J. Bell, K.L. Parker, R.D. Swinford, A.R. Hoffman, T. Maneatis, B. Lippe. Long-term safety of recombinant human growth hormone in children. J Clin Endocrinol Metab, 95 (2010), pp. 167-177. 
39. P. Wilton, A.F. Mattsson, F. Darendeliler. Growth hormone treatment in children is not associated with an increase in the incidence of cancer: Experience from KIGS (Pfizer International Growth Database). J Pediatr, 157 (2010), pp. 265-270.

40. C.A. Sklar, A.C. Mertens, P. Mitby, G. Occhiogrosso, J. Qin, G. Heller, et al. Risk of disease recurrence and second neoplasms in survivors of childhood cancer treated with growth hormone: A report from the Childhood Cancer Survivor Study. J Clin Endocrinol Metab, 87 (2002), pp. 3136-3141.

41. B. Ergun-Longmire, A.C. Mertens, P. Mitby, J. Qin, G. Heller, W. Shi, et al. Growth hormone treatment and risk of second neoplasms in the childhood cancer survivor. J Clin Endocrinol Metab, 91 (2006), pp. 3494-3498.

42. J. Svensson, B.A. Bengtsson. Safety aspects of GH replacement. Eur J Endocrinol, 161 (Suppl 1) (2009), pp. S65-S74.

43. J.C. Carel, E. Ecosse, F. Landier, D. Meguellati-Hakkas, F. Kaguelidou, G. Rey, et al. Long-term mortality after recombinant growth hormone treatment for isolated growth hormone deficiency or childhood short stature: Preliminary report of the French SAGhE study. J Clin Endocrinol Metab, 97 (2012), pp. 416-425.

44. C.J. Child, A.G. Zimmermann, W.W. Woodmansee, D.M. Green, J.J. Li, H. Jung, et al. Assessment of primary cancers in GH-treated adult hypopituitary patients: An analysis from the Hypopituitary Control and Complications Study. Eur J Endocrinol, 165 (2011), pp. 217-223.

45. C.C. Van Bunderen, C. van Nieuwpoort, L.I. Arwert, M.W. Heymans, A.A. Franken, H.P. Koppeschaar, et al. Does growth hormone replacement therapy reduce mortality in adults with growth hormone deficiency? Data from the Dutch National Registry of Growth Hormone Treatment in adults. J Clin Endocrinol Metab, 96 (2011), pp. 3135-3159.

46. P. Wilton, R. Gaillard, B. Saller, M. Koltowska-Häggström, A. Mattsson. Cause-specific mortality in patients from the KIMS database and associations with IGF-I standard deviation scores (SDS). Growth Horm IGF Res, 18 (2008), p. S3.

47. R.C. Gaillard, A.F. Mattsson, A.C. Åkerblad, B.Å. Bengtsson, J. Cara, U. Feldt-Rasmussen, et al. Overall and cause-specific mortality in GH-deficient adults on GH replacement. Eur J Endocrinol, 166 (2012), pp. 10691077.

48. J.J. Díez, P. Iglesias. Optimización del tratamiento médico de la acromegalia. Med Clin (Barc), 140 (2013), pp. 360-365.

49. B.M. Biller, H.J. Ji, H. Ahn, C. Savoy, E.C. Siepl, V. Popovic, et al. Effects of once-weekly sustained-release growth hormone: A double-blind, placebo-controlled study in adult growth hormone deficiency. J Clin Endocrinol Metab, 96 (2011), pp. 1718-1726 